\title{
Protective effects of fucoidan against hyperoxic lung injury via the ERK signaling pathway
}

\author{
MINGHAO NIE, YAN WANG, YANHONG LU, YING YUAN, YINGYING LIU and XIURONG LI \\ Department of Pathology, Heilongjiang Provincial Hospital, Harbin, Heilongjiang 150036, P.R. China
}

Received June 2, 2016; Accepted May 8, 2017

DOI: $10.3892 / \mathrm{mmr} .2017 .8022$

\begin{abstract}
High oxygen mechanical ventilation is widely used to treat various lung diseases; however, it may result in hyperoxia, which induces inflammation and lung injury. Fucoidan is an extract of the seaweed Fucus vesiculosus, which has previously been reported to exert effects against diabetic nephropathy. The present study is the first, to the best of our knowledge, to investigate the protective effects of fucoidan against hyperoxic lung injury. Balb/c mice were ventilated with $100 \%$ oxygen, with or without the atomization inhalation of fucoidan, for $36 \mathrm{~h}$. Hyperoxia reduced the body weight and increased the relative lung weight of the mice. In addition, cell quantity and differentiation were determined using a hemocytometer, hyperoxia increased the total number of cells, and the number of macrophages, neutrophils and lymphocytes in the bronchoalveolar lavage fluid. Reverse transcription-quantitative polymerase chain reaction (RT-qPCR) demonstrated that hyperoxia also increased the mRNA expression levels of cluster of differentiation (CD)68, F4/80, CD64 and CD19 in lung tissue, and induced lung morphological alterations. Furthermore, western blotting assay demonstrated that hyperoxia increased the expression levels of interleukin (IL)-1, IL-6 and tumor necrosis factor (TNF)- $\alpha$, and the phosphorylation of extracellular signal-regulated kinase (ERK)1/2. Conversely, hyperoxia-induced inflammation and morphological alterations were significantly attenuated in the mice treated with fucoidan. Atomization inhalation of fucoidan also reduced the hyperoxia-induced expression of IL-1, IL- 6 and TNF- $\alpha$, and the phosphorylation of ERK1/2. These findings suggested that fucoidan may attenuate hyperoxic lung injury via the ERK1/2 signaling pathway.
\end{abstract}

Correspondence to: Professor Xiurong Li, Department of Pathology, Heilongjiang Provincial Hospital, 82 Zhongshan Road, Harbin, Heilongjiang 150036, P.R. China

E-mail: lixiurong8817@sina.com

Key words: fucoidan, hyperoxic lung injury, interleukin-1, interleukin-6, tumor necrosis factor- $\alpha$, extracellular signal-regulated kinase $1 / 2$

\section{Introduction}

High oxygen mechanical ventilation is widely used as a clinical therapy to treat various diseases (1), including conditions associated with serious respiratory failure, such as hypoxemia and acute respiratory distress syndrome (ARDS) (2). However, as suggested in a previous report (3), exposure to high levels of oxygen for prolonged periods of time may lead to inflammation and lung injury (4). Reactive oxygen species (ROS) are generated under hyperoxic conditions and result in oxidative damage and inflammatory response in the injured lung tissue (5). Inflammatory cells accumulating in the lung also generate ROS (6). Currently, antioxidants and antiallergic drugs are used to protect the lung against hyperoxic lung injury $(7,8)$. However, these drugs often bring side effects. Therefore, the development of a therapeutic strategy that alleviates hyperoxia-induced lung injury without side effects is required.

Interest in the biological activities of marine organisms has intensified in the last few years (9). Fucoidan, the chemical structure of which is presented in Fig. 1A, is an extract of the seaweed Fucus vesiculosus, which has been widely investigated and has been reported to serve an important role in cancer (10) and inflammation (11). Fucoidan exhibits antioxidant, anticancer and anti-inflammatory activities (12), and our previous study demonstrated its effects against diabetic nephropathy (13). The present study aimed to determine whether fucoidan may attenuate or prevent hyperoxia-induced lung injury.

Activation of extracellular signal-regulated kinase (ERK) $1 / 2$ has been reported to be associated with defense signaling against injury (14). High oxygen mechanical ventilation may induce the phosphorylation of ERK1/2, and the secretion of chemokines and cytokines, such as interleukin (IL)-1, IL-6 and tumor necrosis factor (TNF)- $\alpha$ (15), finally leading to inflammatory cell infiltration (16) and lung injury. The present study indicated that hyperoxia induced inflammation and morphological alterations in mouse lung tissue. Conversely, atomization inhalation of fucoidan reduced hyperoxia-induced inflammation, morphological alterations, the expression levels of IL-1, IL- 6 and TNF- $\alpha$, and the phosphorylation of ERK1/2. These findings suggest that fucoidan may attenuate hyperoxic lung injury via the ERK1/2 signaling pathway, thus indicating a novel therapeutic strategy for the alleviation of hyperoxia-induced lung injury. 


\section{Materials and methods}

Animals. The present study was approved by the Ethics Committee of Harbin Medical University (Harbin, China). The experiments were conducted at the Animal Experimental Center of Harbin Medical University in accordance with the Animal Experiment Guidelines from Harbin Medical University. Balb/c mice, not limited to gender (male/female ratio was 1:1), were housed in a controlled environment, with a temperature of $24 \pm 1^{\circ} \mathrm{C}$, and a 12-h light/dark cycle. Briefly, $\mathrm{Balb} / \mathrm{c}$ mice (age, 6-8 weeks; Institute of Laboratory Animal Sciences, Shanghai, China) were fed normal chow and water ad libitum. The mice ( $\mathrm{n}=5-6$ per group) were ventilated with $100 \%$ oxygen, with or without atomization inhalation of fucoidan (100 $\mu \mathrm{g} / \mathrm{ml}$; Kanehide Bio Co., Ltd., Okinawa, Japan), for $36 \mathrm{~h}$, according to previous studies $(17,18)$. Untreated mice were used as control. Following exposure to $100 \%$ oxygen, the body weight and relative lung weight of Balb/c mice were measured before and after sacrifice respectively, and lung tissue and bronchoalveolar lavage fluid (BALF) samples were collected for further experimentation.

Preparation of BALF. The lungs were washed with $0.5 \mathrm{ml}$ sterile saline four times through a $21 \mathrm{G}$ flat syringe needle cannulated $0.7 \mathrm{~cm}$ into the trachea. The BALF recovered from each mouse was subsequently used for quantitative cell counting. Cell quantity and differentiation were determined using a hemocytometer (Countess ${ }^{\circledR}$ II Automated Cell Counter; AMQAX1000; Thermo Fisher Scientific, Inc., Waltham, MA, USA) for $>500$ cells placed on cytocentrifuge slides and stained with Wright-Giemsa (19). The total cell number was counted by the Automated Cell Counter, and the macrophages, neutrophils and lymphocytes with Giemsa staining were confirmed and counted under a microscope (Olympus Corporation, Tokyo, Japan) at a magnification of $\mathrm{x} 100$. A $100 \mathrm{ml}$ aliquot of BALF was collected, after total cell counting, the remainder was immediately centrifuged at $1,000 \mathrm{x}$ g for $10 \mathrm{~min}$. Macrophages were isolated from the BALF. The BALF supernatants were stored at $-80^{\circ} \mathrm{C}$ for cytokine and chemokine analyses.

Reverse transcription-quantitative polymerase chain reaction $(R T-q P C R)$. Total RNA was extracted from the lung tissue by TRIzol (Sigma-Aldrich; Merck KGaA, Darmstadt, Germany) and the relative mRNA expression levels of cluster of differentiation (CD)68, F4/80, CD64 and CD19 were normalized to GAPDH. The following primers were used: CD68, forward 5'-CATCAGAGCCCGAGTACAGTCTACC-3', reverse 5'-AAT TCTGCGCCATGAATGTCC-3'; F4/80, forward 5'-GAGATT GTGGAAGCATCCGAGAC-3', reverse, 5'-GATGACTGT ACCCACATGGCTGA-3'; CD64, forward 5'-CTTCTCCTT CTATGTGGGCAGT-3', reverse 5'-GCTACCTCGCACCAG TATGAT-3'; CD19, forward 5'-CCACAAAGTCCCAGCTGA AT-3', reverse, 5'-GGGGTCCCAGATTTCAAAGT-3'; and GAPDH, forward 5'-GCACCGTCAAGGCTGAGAAC-3' and reverse 5'-TGGTGAAGACGCCAGTGGA-3'. A Transcriptor First Strand cDNA Synthesis kit (Roche Applied Science, Madison, WI, USA) was used for the reverse transcription. qPCR was performed using the ABI 7300 Fast Real-Time PCR system (Applied Biosystems; Thermo Fisher Scientific, Inc.) and the thermocycling conditions were: Holding stage $95^{\circ} \mathrm{C}$ for $30 \mathrm{sec}, 1 \mathrm{cycle}$ and cycling stage at $95^{\circ} \mathrm{C}$ for $3 \mathrm{sec}$ and 40 cycles at $60^{\circ} \mathrm{C}$ for $31 \mathrm{sec}$. The $30 \mathrm{~min}$ electrophoresis with agarose gel with ethidium bromide (Sigma-Aldrich, Merck $\mathrm{KGaA}$ ). Every experiment was repeated 3 times. As described in reference (20), the image analysis was performed using the Image $\mathrm{Lab}^{\mathrm{TM}}$ software (version 2.1; Bio-Rad Laboratories, Inc., Hercules, CA, USA). PCR results were analyzed by $2^{-\Delta \Delta C q}$ method (21).

Morphometric analysis. As previously described, (22), lung tissues were fixed in formalin (Sigma-Aldrich; Merck KGaA), cleared in xylene (Sigma-Aldrich; Merck KGaA) and embedded in paraffin. The paraffin-embedded lung tissue samples were examined by conventional light microscopic examination: $5 \mu \mathrm{m}$ sections were stained with hematoxylin and eosin, and were assessed in a blinded manner. An automatic microscope (Provis AX-70) with a camera (Olympus Corporation, Tokyo, Japan) was used to capture the microscopic images of the lung samples. The morphometric analysis was performed using ImageJ software version 1.60 (National Institutes of Health, Bethesda, MD, USA) (23).

Enzyme-linked immunosorbent assay (ELISA). BALF and homogenized lung tissue were collected and underwent IL-1, IL- 6 and TNF- $\alpha$ assays using respective mouse ELISA kits (RAB0275, RAB0309 and GERPN2718; Merck KGaA). The ELISA plates were incubated with $100 \mu 1$ capture antibody per well at $4^{\circ} \mathrm{C}$ overnight. After appropriate washing by the washing buffer from the kits, $200 \mu \mathrm{l}$ assay dilution buffer was added per well for blocking at room temperature for $1 \mathrm{~h}$. Serial dilutions of standards and samples were added to each well and were incubated at $4^{\circ} \mathrm{C}$ overnight. Avidin-horseradish peroxidase (HRP) was added following incubation with the detection antibody for $30 \mathrm{~min}$ at room temperature, and the samples were further incubated at room temperature for another $30 \mathrm{~min}$. Subsequently, the 3,3',5,5'-tetramethylbenzidine substrate was added, and following incubation for $15 \mathrm{~min}$ at room temperature, $\mathrm{NH}_{2} \mathrm{SO}_{4}$ was added to terminate the reaction and absorbance was measured at $450 \mathrm{~nm}$ using an ELISA reader (MTP-800 microplate reader; Corona Electric Co., Ltd., Tokyo, Japan).

Western blotting. According to method described by Laemmli (24) for western blotting, electrophoresis was performed using a vertical slab gel containing $12 \%$ polyacrylamide. Lung tissues were ground and lysed with radio-immunoprecipitation assay lysis buffer (Beyotime Institute of Biotechnology, Shanghai, China). The concentration of proteins extracted from lung tissues was determined by Gene Spec III from Hitachi Genetic Systems (MiraiBio Group of Hitachi Solutions America, Ltd., South San Francisco, CA, USA) and $20 \mu \mathrm{g}$ of protein was loaded per gel lane. Proteins were separated by SDS-PAGE and were then electrophoretically transferred to a supported nitrocellulose membrane (Bio-Rad Laboratories, Inc.) using a Semi-Dry Electroblotter for 90 min at $15 \mathrm{~V}$ (Sartorius AG, Göttingen, Germany) (25). The membrane was then treated with Block Ace ${ }^{\mathrm{TM}}(4 \%)$ at $22^{\circ} \mathrm{C}$ for $30 \mathrm{~min}$. The membrane was then incubated with rabbit immunoglobulin (Ig)G anti-ERK1/2 (SAB1305560; 1:1,000), 

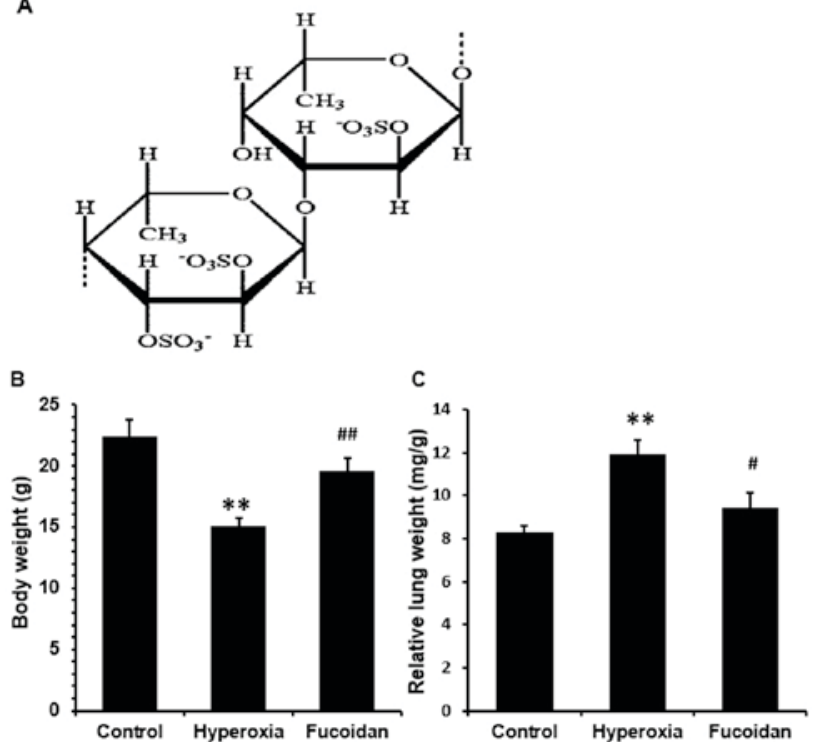

Figure 1. (A) Chemical structure of fucoidan, an extract of the seaweed Fucus vesiculosus. Hyperoxia-induced (B) decreases in body weight and (C) increases in lung weight were attenuated by fucoidan. The Balb/c mice were ventilated with $100 \%$ oxygen, with or without atomization inhalation of fucoidan $(100 \mu \mathrm{g} / \mathrm{ml})$, for $36 \mathrm{~h}$. Hyperoxia significantly reduced mouse body weight and increased relative lung weight, whereas fucoidan reversed the hyperoxia-induced alterations in body weight and relative lung weight. Data are presented as the mean \pm standard deviation ( $\mathrm{n}=5-6$ per group). ${ }^{* *} \mathrm{P}<0.01$, vs. control mice; ${ }^{\#} \mathrm{P}<0.05,{ }^{\# \#} \mathrm{P}<0.01$, vs. hyperoxia-treated mice.

anti-p-ERK1/2 (SAB4301578; 1:1,000) and anti- $\beta$-actin (SAB2100037; 1:1, Sigma-Aldrich; Merck KGaA) in phosphate-buffered saline containing $0.03 \%$ Tween 20 at $22^{\circ} \mathrm{C}$ for $1 \mathrm{~h}$. Following washing in the same buffer, the membrane was incubated with HRP-conjugated goat anti-rabbit IgG (A0545; $20 \mathrm{ng} / \mathrm{ml}$; Sigma-Aldrich; Merck KGaA) at $22^{\circ} \mathrm{C}$ for $30 \mathrm{~min}$. Following further washing, an Enhanced Chemiluminescence Plus Western Blotting Detection system (GE Healthcare Life Sciences, Tokyo, Japan) was used to visualize the blot. ImageJ version $1.48 \mathrm{u}$ (National Institutes of Health) was used for the quantification of western blots.

Statistical analysis. Analyses were performed using SPSS version 19.0 (IBM Corp., Armonk, NY, USA). Results are presented as the mean \pm standard deviation. Each experiment was repeated at least three times. Multiple comparisons between groups were performed using one-way analysis of variance and Dunnett's test. $\mathrm{P}<0.05$ was considered to indicate a statistically significant difference.

\section{Results}

Hyperoxia-induced decreases in body weight and increases in lung weight are attenuated by fucoidan. Balb/c mice were ventilated with $100 \%$ oxygen, with or without atomization inhalation of fucoidan $(100 \mu \mathrm{g} / \mathrm{ml})$, for $36 \mathrm{~h}$. Hyperoxia significantly reduced mouse body weight (Fig. 1B) and increased relative lung weight (Fig. $1 \mathrm{C} ; \mathrm{P}<0.01$ ). Conversely, fucoidan significantly increased body weight $(\mathrm{P}<0.01)$ and reduced relative lung weight $(\mathrm{P}<0.05)$ compared with the hyperoxia group.
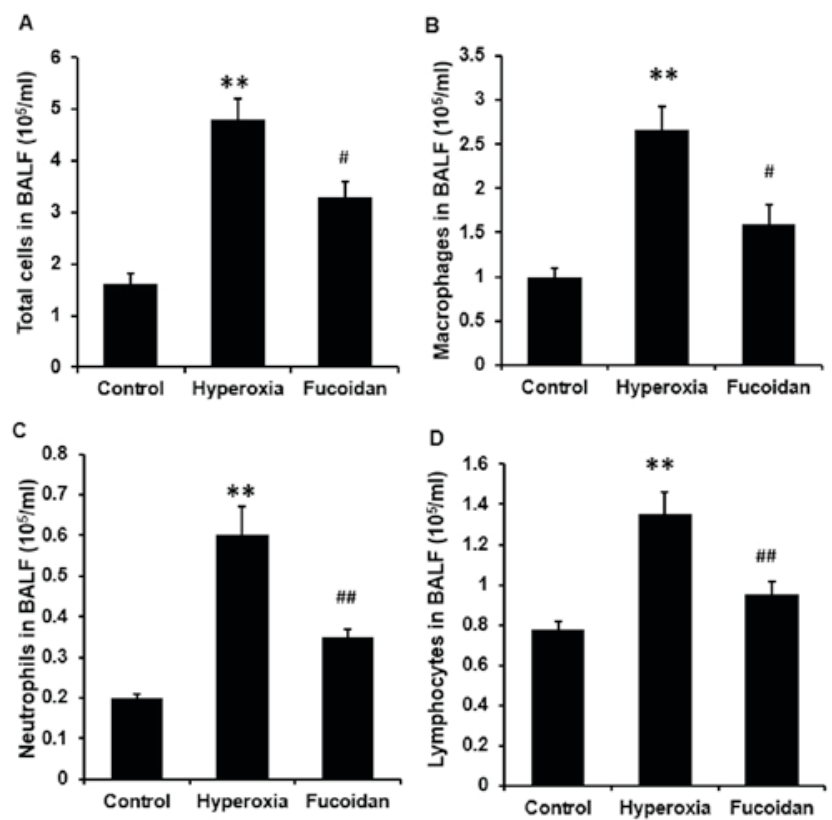

Figure 2. Hyperoxia-induced inflammatory cell infiltration into the BALF is attenuated by fucoidan. Following ventilation with $100 \%$ oxygen, with or without atomization inhalation of fucoidan $(100 \mu \mathrm{g} / \mathrm{ml})$, for $36 \mathrm{~h}$, BALF samples of Balb/c mice were collected. (A) Total cell counts and the number of (B) macrophages, (C) neutrophils and (D) lymphocytes in the BALF were determined on cytocentrifuge slides using a hemocytometer. Hyperoxia significantly increased total cell count, and the number of macrophages, neutrophils and lymphocytes, compared with control mice. The hyperoxia-induced increases in total cell count, and the number of macrophages, neutrophils and lymphocytes, in the BALF were significantly reduced in mice receiving fucoidan. Data are presented as the mean \pm standard deviation ( $n=6$ per group). ${ }^{* *} \mathrm{P}<0.01$, vs. control mice; ${ }^{\#} \mathrm{P}<0.05,{ }^{\# \#} \mathrm{P}<0.01$, vs. hyperoxia-treated mice. BALF, bronchoalveolar lavage fluid.

Hyperoxia-induced inflammatory cell infiltration into the $B A L F$ is attenuated by fucoidan. Following ventilation with $100 \%$ oxygen, with or without atomization inhalation of fucoidan $(100 \mu \mathrm{g} / \mathrm{ml})$, for $36 \mathrm{~h}$, BALF samples of Balb/c mice were collected. Total cell counts, and the number of macrophages, neutrophils and lymphocytes, in the BALF were determined on cytocentrifuge slides using a hemocytometer. Hyperoxia significantly increased total cell count (Fig. 2A), and the number of macrophages (Fig. 2B), neutrophils (Fig. 2C) and lymphocytes (Fig. 2D) compared with the control mice $(\mathrm{P}<0.01)$. The hyperoxia-induced increases in the total cell count, and the number of macrophages, neutrophils and lymphocytes, in the BALF were significantly reduced in mice receiving fucoidan $(\mathrm{P}<0.05)$.

Hyperoxia-induced inflammatory cell infiltration into lung tissue is attenuated by fucoidan. Following ventilation with $100 \%$ oxygen, with or without atomization inhalation of fucoidan $(100 \mu \mathrm{g} / \mathrm{ml})$, for $36 \mathrm{~h}$, lung tissue of Balb/c mice was collected. The mRNA expression levels of CD68, F4/80 (macrophage markers) (Fig. 3A), CD64 (neutrophil marker) and CD19 (lymphocyte marker) (Fig. 3B) were detected in the lung tissue samples. The mRNA expression levels of CD68, F4/80, CD64 and CD19 were significantly increased in lung tissue by hyperoxia. Treatment with fucoidan significantly suppressed hyperoxia-induced mRNA expression $(\mathrm{P}<0.01)$. 
A

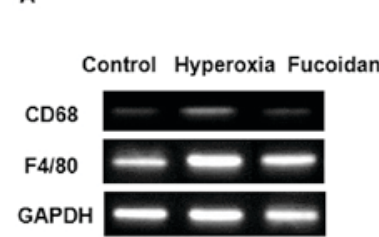

B

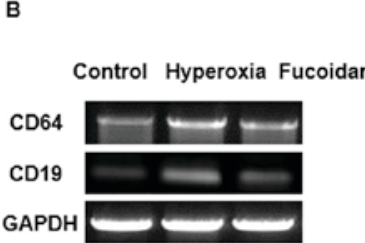

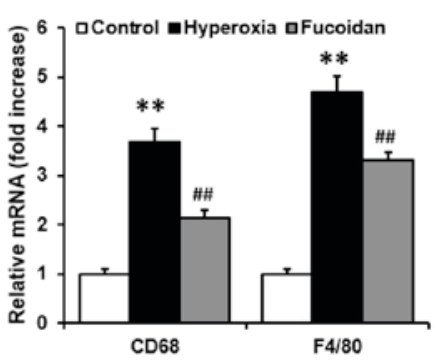

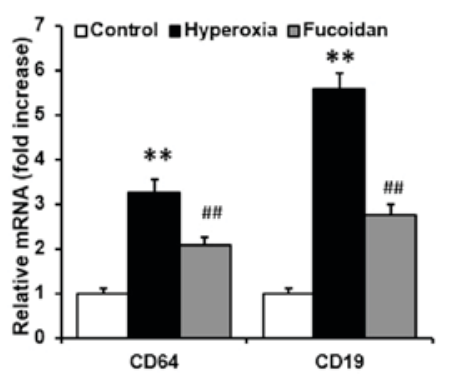

Figure 3. Hyperoxia-induced inflammatory cell infiltration in lung tissue is attenuated by fucoidan. Following ventilation with $100 \%$ oxygen, with or without atomization inhalation of fucoidan $(100 \mu \mathrm{g} / \mathrm{ml})$, for $36 \mathrm{~h}$, lung tissue was collected from Balb/c mice. The mRNA expression levels of (A) CD68, F4/80 (macrophage markers), (B) CD64 (neutrophil marker) and CD19 (lymphocyte marker) were detected in the lung tissue samples. The mRNA expression levels of CD68, F4/80, CD64 and CD19 were significantly increased in lung tissue by hyperoxia. Treatment with fucoidan significantly suppressed hyperoxia-induced mRNA expression. Data are presented as the mean \pm standard deviation ( $\mathrm{n}=6$ per group). ${ }^{* *} \mathrm{P}<0.01$, vs. control mice; ${ }^{\# \#} \mathrm{P}<0.01$, vs. hyperoxia-treated mice. $\mathrm{CD}$, cluster of differentiation.
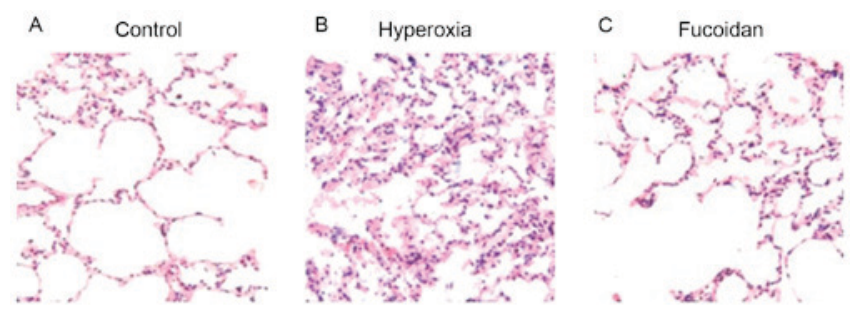

Figure 4. Hyperoxia-induced lung morphological alterations are attenuated by fucoidan. Balb/c mice were ventilated with $100 \%$ oxygen, with or without atomization inhalation of fucoidan $(100 \mu \mathrm{g} / \mathrm{ml})$, for $36 \mathrm{~h}$. Lung morphological alterations were detected in the (A) control, (B) hyperoxia and (C) fucoidan groups. Hyperoxia increased alveolar wall thickness and induced inflammatory cell infiltration into the lung tissue. Hyperoxia-induced morphological alterations were attenuated by fucoidan treatment $(n=3$ per group). Magnification, x200.

Hyperoxia-induced lung morphological alterations are attenuated by fucoidan. Balb/c mice were ventilated with $100 \%$ oxygen, with or without atomization inhalation of fucoidan $(100 \mu \mathrm{g} / \mathrm{ml})$, for $36 \mathrm{~h}$. Lung morphological alterations were detected. Hyperoxia increased the thickness of the alveolar walls and induced inflammatory cell infiltration into the lung tissue. Conversely, the hyperoxia-induced morphological alterations were attenuated by fucoidan treatment (Fig. 4; magnification, $\mathrm{x} 200$ ).

Hyperoxia-induced increases in the expression of inflammatory factors in the BALF and lung tissue are attenuated by fucoidan. Balb/c mice were ventilated with $100 \%$ oxygen, with

or without atomization inhalation of fucoidan $(100 \mu \mathrm{g} / \mathrm{ml})$, for $36 \mathrm{~h}$. BALF and lung tissue samples were then harvested. The expression levels of IL-1, IL- 6 and TNF- $\alpha$ in the BALF (Fig. 5A) and lung tissue (Fig. 5B) were significantly increased by hyperoxia $(\mathrm{P}<0.01)$. Hyperoxia-induced increases in the expression levels of IL-1, IL-6 and TNF- $\alpha$ in the BALF and lung tissue were significantly reduced following treatment with fucoidan $(\mathrm{P}<0.05)$.

Hyperoxia-induced phosphorylation of ERK1/2 is attenuated by fucoidan. Balb/c mice were ventilated with $100 \%$ oxygen, with or without atomization inhalation of fucoidan $(100 \mu \mathrm{g} / \mathrm{ml})$, for $36 \mathrm{~h}$. Lung tissue samples were then harvested. Hyperoxia significantly increased phosphorylation of ERK1/2 in lung tissue (Fig. 6). However, the hyperoxia-induced phosphorylation of ERK1/2 in the lung tissue was significantly reduced following treatment with fucoidan $(\mathrm{P}<0.01)$.

\section{Discussion}

In recent years, high oxygen mechanical ventilation has been widely used as a clinical therapy to treat various diseases (1), including conditions associated with serious respiratory failure, such as hypoxemia and ARDS (2). However, as suggested in a previous study (3), and clinically (26), exposure to high levels of oxygen for prolonged periods of time may lead to inflammation and lung injury (4). Therefore, a therapeutic strategy that alleviates hyperoxia-induced lung injury is required.

Interest in the biological activities of marine organisms has intensified (9). Fucoidan, which is an extract of the seaweed Fucus vesiculosus, has been widely investigated and has been reported to serve an important role in cancer (10) and inflammation (11). Fucoidan is able to suppress various inflammatory cytokines, including IL-1 $\beta$, TNF- $\alpha$, interferon- $\gamma$ and cyclooxygenase-2 (11). Fucoidan is an antioxidant, anticancer and anti-inflammatory agent (12), which also exerts effects against diabetic nephropathy (13). The present study investigated the protective effects of fucoidan against hyperoxia-induced lung injury. Prior to the present study, we performed a dose-dependent study using 1, 10, 100 and $1,000 \mu \mathrm{g} / \mathrm{ml}$ fucoidan; significant alterations occurred when $\geq 100 \mu \mathrm{g} / \mathrm{ml}$ fucoidan was used; therefore, $100 \mu \mathrm{g} / \mathrm{ml}$ was used as the standard concentration in the present study.

The present study demonstrated that hyperoxia significantly increased total cell count, and the number of macrophages, neutrophils and lymphocytes in the BALF. In addition, hyperoxia significantly increased the mRNA expression levels of CD68 and F4/80 (macrophage markers), CD64 (neutrophil marker) and CD19 (lymphocyte marker), and induced increases in alveolar wall thickness and inflammatory cell infiltration into the lung tissue. These results are consistent with those of a previous study, which suggested that hyperoxia may recruit inflammatory cells, such as macrophages, lymphocytes and neutrophils (27). Fucoidan significantly reduced hyperoxia-induced inflammatory cell infiltration into the BALF and lung tissue, and attenuated the morphological alterations. Furthermore, normal mice were treated with fucoidan only; no significant differences were detected between the control and fucoidan-treated normal mice (data not shown), strongly indicating that fucoidan did not exhibit cytotoxicity. 


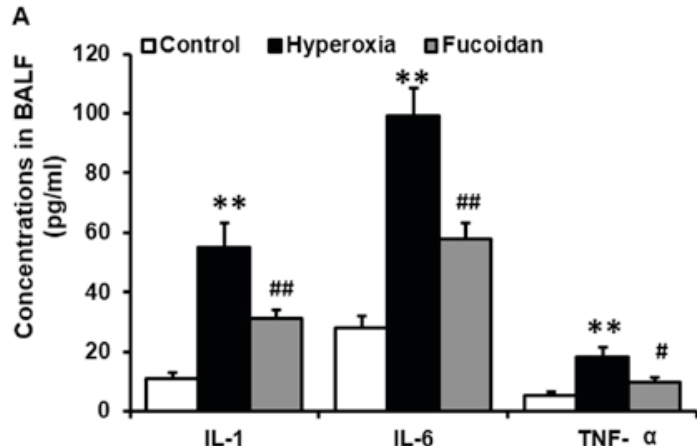

B

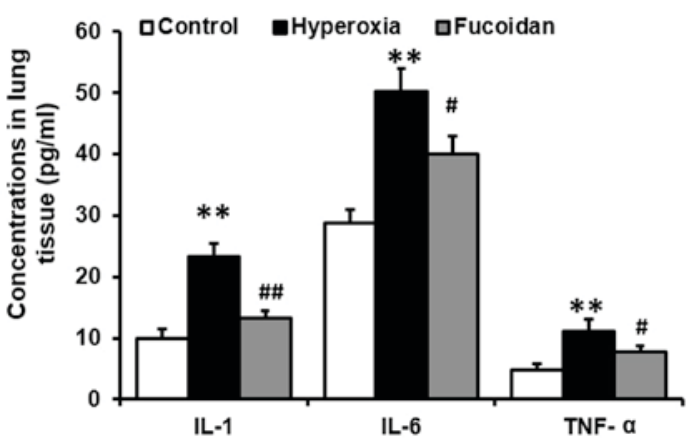

Figure 5. Hyperoxia-induced increases in the expression levels of inflammatory factors in the (A) BALF and (B) lung tissue are attenuated by fucoidan. $\mathrm{Balb} / \mathrm{c}$ mice were ventilated with $100 \%$ oxygen, with or without atomization inhalation of fucoidan $(100 \mu \mathrm{g} / \mathrm{ml})$, for $36 \mathrm{~h}$. BALF and lung tissue samples were then harvested. The expression levels of IL-1, IL- 6 and TNF- $\alpha$ in the BALF and lung tissue samples were significantly increased by hyperoxia, whereas the expression levels of IL-1, IL-6 and TNF- $\alpha$ were significantly reduced in the BALF and lung tissue following treatment with Fucoidan. Data are presented as the mean \pm standard deviation ( $n=6$ per group). ${ }^{* *} \mathrm{P}<0.01$ vs. control mice; ${ }^{\#} \mathrm{P}<0.05,{ }^{\# \prime} \mathrm{P}<0.01$, vs. hyperoxia-treated mice. BALF, bronchoalveolar lavage fluid; IL, interleukin; TNF- $\alpha$, tumor necrosis factor- $\alpha$.
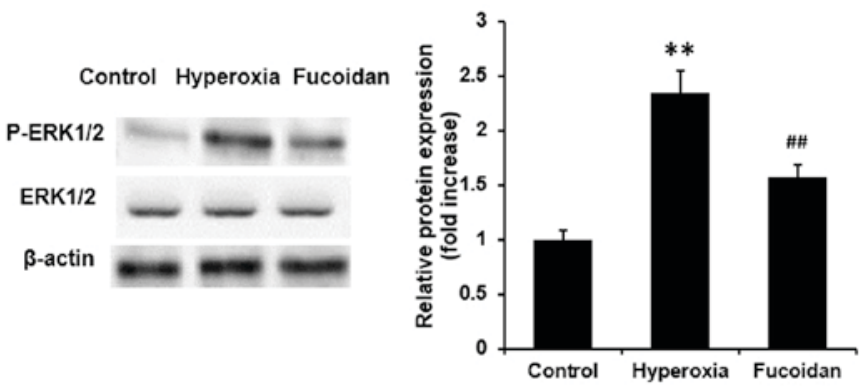

Figure 6. Hyperoxia-induced phosphorylation of ERK1/2 is attenuated by fucoidan. Balb/c mice were ventilated with $100 \%$ oxygen, with or without atomization inhalation of fucoidan $(100 \mu \mathrm{g} / \mathrm{ml})$, for $36 \mathrm{~h}$. Lung tissue samples were then harvested. Hyperoxia significantly increased phosphorylation of ERK1/2 in lung tissue. However, hyperoxia-induced phosphorylation of ERK1/2 in the lung tissue was significantly reduced following treatment with fucoidan. Data are presented as the mean \pm standard deviation $(n=5$ per group). ${ }^{* *} \mathrm{P}<0.01$, vs. control mice; ${ }^{\# \#} \mathrm{P}<0.01$, vs. hyperoxia-treated mice ERK1/2, extracellular signal-regulated kinase 1/2; P-, phosphorylated.

High oxygen mechanical ventilation may lead to lung injury, and secretion of chemokines and cytokines, such as IL-1 (28), IL-6 (29) and TNF- $\alpha$ (30), thus resulting in inflammatory cell infiltration $(15,16)$. It has previously been reported that IL-1 and IL- 6 are associated with antiand proinflammatory responses, through the regulation

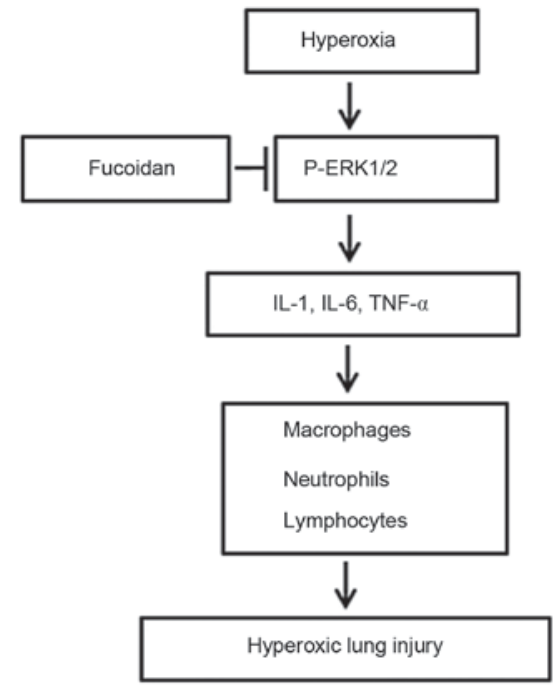

Figure 7. Protective mechanism of fucoidan in hyperoxia-induced lung injury. Hyperoxia induced phosphorylation of ERK1/2, increased the expression levels of IL-1, IL-6 and TNF- $\alpha$, and induced lung morphological alterations and inflammatory cell infiltration. Hyperoxia-induced effects were attenuated following treatment with fucoidan. ERK1/2, extracellular signal-regulated kinase $1 / 2$; P-, phosphorylated; IL, interleukin; TNF- $\alpha$, tumor necrosis factor- $\alpha$.

of leukocyte apoptosis and function, and may beneficially regulate neutrophil migration and adhesion (31). Elevated levels of IL-1, IL- 6 and TNF- $\alpha$ have been demonstrated as biological markers in numerous models of lung injury $(32,33)$. To improve understanding regarding hyperoxia-induced lung injury, the expression levels of IL-1, IL-6 and TNF- $\alpha$ in the BALF and lung tissue were examined in the present study. Hyperoxia significantly increased the expression levels of IL-1, IL-6 and TNF- $\alpha$ in the BALF and lung tissue, whereas hyperoxia-induced IL-1, IL- 6 and TNF- $\alpha$ expression was significantly attenuated in the BALF and lung tissue following treatment with fucoidan.

It has also been demonstrated that activation of the ERK1/2 pathway may be involved in defense signaling against oxidative injury (14). The ERK1/2 pathway is a signaling cascade that is involved in protection from damage, the activation of which is generally hypothesized to mediate cell survival (34). Secretion of chemokines and cytokines, such as IL-1, IL-6 and TNF- $\alpha$, are associated with the phosphorylation of ERK1/2 (15). To better understand the underlying mechanism, the present study examined the phosphorylation of ERK1/2. Hyperoxia significantly induced phosphorylation of ERK1/2 in lung tissue; however, hyperoxia-induced phosphorylation of ERK1/2 was significantly reduced following treatment with fucoidan.

The protective mechanism of fucoidan in hyperoxia-induced lung injury is presented in Fig. 7. Briefly, hyperoxia induced phosphorylation of ERK1/2, increased the expression levels of IL-1, IL-6 and TNF- $\alpha$, and induced lung morphological alterations and inflammation. However, these effects were attenuated following treatment with fucoidan.

In conclusion, to the best of our knowledge, the present study is the first to demonstrate the crucial role of fucoidan against hyperoxia-induced lung injury via the ERK1/2 signaling pathway. These results suggested a novel therapeutic strategy for the alleviation of hyperoxia-induced lung injury. 


\section{References}

1. Xu Y, Tian $\mathrm{Z}$ and Xie P: Targeting complement anaphylatoxin C5a receptor in hyperoxic lung injury in mice. Mol Med Rep 10: 1786-1792, 2014

2. Vitacca M, Bianchi L, Bazza A and Clini EM: Advanced COPD patients under home mechanical ventilation and/or long term oxygen therapy: Italian healthcare costs. Monaldi Arch Chest Dis 75: 207-214, 2011.

3. Panikkanvalappil SR, James M, Hira SM, Mobley J, Jilling T, Ambalavanan N and El-Sayed MA: Hyperoxia induces intracellular acidification in neonatal mouse lung fibroblasts: Real-time investigation using plasmonically enhanced Raman spectroscopy. J Am Chem Soc 138: 3779-3788, 2016.

4. Vosdoganes P, Lim R, Koulaeva E, Chan ST, Acharya R, Moss TJ and Wallace EM: Human amnion epithelial cells modulate hyperoxia-induced neonatal lung injury in mice. Cytotherapy 15: 1021-1029, 2013

5. George CL, Fantuzzi G, Bursten S, Leer L and Abraham E: Effects of lisofylline on hyperoxia-induced lung injury. Am J Physiol 276: L776-L785, 1999.

6. Kallet RH and Matthay MA: Hyperoxic acute lung injury. Respir Care 58: 123-141, 2013.

7. Johnson-Varghese L, Brodsky N and Bhandari V: Effect of antioxidants on apoptosis and cytokine release in fetal rat Type II pneumocytes exposed to hyperoxia and nitric oxide. Cytokine 28 $10-16,2004$

8. Fukuhara K, Nakashima T, Abe M, Masuda T, Hamada H, Iwamoto H, Fujitaka K, Kohno N and Hattori N: Suplatast tosilate protects the lung against hyperoxic lung injury by scavenging hydroxyl radicals. Free Radic Biol Med 106: 1-9, 2017.

9. Xie P, Fujii I, Zhao J, Shinohara M and Matsukura M: A novel polysaccharide compound derived from algae extracts protects retinal pigment epithelial cells from high glucose-induced oxidative damage in vitro. Biol Pharm Bull 35: 1447-1453, 2012.

10. Kasai A, Arafuka S, Koshiba N, Takahashi D and Toshima K: Systematic synthesis of low-molecular weight fucoidan derivatives and their effect on cancer cells. Org Biomol Chem 13 10556-10568, 2015.

11. Fukahori S, Yano H, Akiba J, Ogasawara S, Momosaki S, Sanada S, Kuratomi K, Ishizaki Y, Moriya F, Yagi M and Kojiro M: Fucoidan, a major component of brown seaweed, prohibits the growth of human cancer cell lines in vitro. Mol Med Rep 1: 537-542, 2008.

12. Li X, Zhao H, Wang Q, Liang H and Jiang X: Fucoidan protects ARPE-19 cells from oxidative stress via normalization of reactive oxygen species generation through the $\mathrm{Ca}^{2+}$-dependent ERK signaling pathway. Mol Med Rep 11: 3746-3752, 2015.

13. Wang Y, Nie M, Lu Y, Wang R, Li J, Yang B, Xia M, Zhang H and Li X: Fucoidan exerts protective effects against diabetic nephropathy related to spontaneous diabetes through the NF- $\mathrm{BB}$ signaling pathway in vivo and in vitro. Int $\mathbf{J}$ Mol Med 35: 1067-1073, 2015 .

14. Glotin AL, Calipel A, Brossas JY, Faussat AM, Tréton J and Mascarelli F: Sustained versus transient ERK1/2 signaling underlies the anti- and proapoptotic effects of oxidative stress in human RPE cells. Invest Ophthalmol Vis Sci 47: 4614-4623, 2006.

15. Pushparaj PN, Tay HK, Wang CC, Hong W and Melendez AJ: VAMP8 is essential in anaphylatoxin-induced degranulation, TNF-alpha secretion, peritonitis, and systemic inflammation. J Immunol 183: 1413-1418, 2009.

16. Davies J, Karmouty-Quintana H, Le TT, Chen NY, Weng T, Luo F, Molina J, Moorthy B and Blackburn MR: Adenosine promotes vascular barrier function in hyperoxic lung injury. Physiol Rep 2: pii: e12155, 2014.

17. Mikawa K, Nishina K, Maekawa N and Obara H: Attenuation of hyperoxic lung injury in rabbits with superoxide dismutase: Effects on inflammatory mediators. Acta Anaesthesiol Scand 39: 317-322, 1995.
18. Takao Y, Mikawa K, Nishina K, Maekawa N and Obara H: Lidocaine attenuates hyperoxic lung injury in rabbits. Acta Anaesthesiol Scand 40: 318-325, 1996.

19. Dolinay T, Wu W, Kaminski N, Ifedigbo E, Kaynar AM, Szilasi M, Watkins SC, Ryter SW, Hoetzel A and Choi AM: Mitogen-activated protein kinases regulate susceptibility to ventilator-induced lung injury. PLoS One 3: e1601, 2008.

20. Villarreal Camacho JL, Mendoza Torres E, Cadena C, Prieto J, Varela Prieto LL and Villanueva Torregroza DA: The use of factorial design, image analysis, and an efficiency calculation for multiplex PCR optimization. J Clin Lab Anal 27: 249-252, 2013.

21. Livak KJ and Schmittgen TD: Analysis of relative gene expression data using real-time quantitative PCR and the 2(-Delta Delta C(T)) method. Methods 25: 402-408, 2001.

22. Jiang X, Ma Y, Yu J, Li H and Xie F: Protective effect of C4a against hyperoxic lung injury via a macrophage-dependent but not a neutrophil/lymphocyte-dependent signaling pathway. Mol Med Rep 13: 1250-1256, 2016.

23. Runzi M, Raptopoulos V, Saluja AK, Kaiser AM, Nishino H, Gerdes D and Steer ML: Evaluation of necrotizing pancreatitis in the opossum by dynamic contrast-enhanced computed tomography: Correlation between radiographic and morphologic changes. J Am Coll Surg 180: 673-682, 1995.

24. Laemmli UK: Cleavage of structural proteins during the assembly of the head of bacteriophage T4. Nature 227: 680-685, 1970.

25. Tsuruta T, Yamamoto T, Matsubara S, Nagasawa S, Tanase S, Tanaka J, Takagi K and Kambara T: Novel function of C4a anaphylatoxin. Release from monocytes of protein which inhibits monocyte chemotaxis. Am J Pathol 142: 1848-1857, 1993.

26. Matsubara S, Yamamoto T, Tsuruta T, Takagi K and Kambara T: Complement C4-derived monocyte-directed chemotaxis inhibitory factor. A molecular mechanism to cause polymorphonuclear leukocyte-predominant infiltration in rheumatoid arthritis synovial cavities. Am J Pathol 138: 1279-1291, 1991.

27. Janssen WJ, Barthel L, Muldrow A, Oberley-Deegan RE, Kearns MT, Jakubzick C and Henson PM: Fas determines differential fates of resident and recruited macrophages during resolution of acute lung injury. Am J Respir Crit Care Med 184: 547-560, 2011.

28. Zhao Y, Xu H, Yu W and Xie BD: Complement anaphylatoxin C4a inhibits C5a-induced neointima formation following arterial injury. Mol Med Rep 10: 45-52. 2014.

29. Jones SA: Directing transition from innate to acquired immunity: Defining a role for IL-6. J Immunol 175: 3463-3468, 2005.

30. Bao JP, Jiang LF, Li J, Chen WP, Hu PF and Wu LD: Visceral adipose tissue-derived serine protease inhibitor inhibits interleukin-1 $\beta$-induced catabolic and inflammatory responses in murine chondrocytes. Mol Med Rep 10: 2191-2197, 2014.

31. Wolters PJ, Wray C, Sutherland RE, Kim SS, Koff J, Mao Y and Frank JA: Neutrophil-derived IL-6 limits alveolar barrier disruption in experimental ventilator-induced lung injury. J Immunol 182: 8056-8062, 2009.

32. Frank JA, Parsons PE and Matthay MA: Pathogenetic significance of biological markers of ventilator-associated lung injury in experimental and clinical studies. Chest 130: 1906-1914, 2006.

33. Piguet PF, Collart MA, Grau GE, Sappino AP and Vassalli P: Requirement of tumour necrosis factor for development of silica-induced pulmonary fibrosis. Nature 344: 245-247, 1990.

34. Guillonneau X, Régnier-Ricard F, Dupuis C, Courtois Y and Mascarelli F: Paracrine effects of phosphorylated and excreted FGF1 by retinal pigmented epithelial cells. Growth Factors 15: 95-112, 1998. 